\title{
Intraoperative monitoring of cerebral blood flow by laser speckle contrast analysis
}

\author{
Nils Hecht, M.D., ${ }^{1,2}$ Johannes WoitziK, M.D., ${ }^{1}$ Jens P. Dreier, M.D., ${ }^{2}$ \\ ANd Peter Vajkoczy, M.D. ${ }^{1,2}$ \\ ${ }^{1}$ Department of Neurosurgery, and ${ }^{2}$ Center for Stroke Research Berlin, Charité-Universitätsmedizin \\ Berlin, Germany
}

\begin{abstract}
Object. Currently, reliable low-cost and noninvasive techniques to assess cerebral perfusion in the operating room are not available. The authors report on their first clinical experience with laser speckle contrast analysis (LASCA) as a complementary imaging tool for the noninvasive and dynamic assessment of cerebral blood flow (CBF) during neurovascular surgery. The purpose of this preliminary study was to address the general feasibility of LASCA in terms of handling and image quality and to provide an example of its clinical implications.

Methods. Laser speckle contrast analysis was performed in patients undergoing cerebral revascularization procedures for the treatment of hemodynamic compromise and complex aneurysms. The portable LASCA device was centered over the surgical field, and continuous 5-minute recordings of relative CBF were obtained. In the case of flow augmentation for hemodynamic compromise, $\mathrm{CBF}$ monitoring was performed before and after completion of the anastomosis. In the case of flow replacement for parent artery sacrifice, CBF monitoring was performed during consecutive 30-second test occlusions of the radial artery graft after proximal internal carotid artery sacrifice and the subsequent initiation of blood flow through the bypass.

Results. In all cases, the authors achieved good visualization of relative CBF in addition to flow imaging in both the bypass graft and the cortical vasculature. During a sudden CBF decrease after test occlusion of the radial artery graft and subsequent flow initiation through the bypass, LASCA allowed immediate visualization and measurement of relative CBF in excellent spatiotemporal resolution.

Conclusions. In this study LASCA offered noninvasive and rapid intraoperative assessment of relative CBF,
\end{abstract} which can be used for optimizing neurovascular procedures.(DOI: 10.3171/2009.8.FOCUS09148)

\section{KEY WoRDS - cerebral blood flow - extracranial-intracranial bypass surgery • intraoperative cortical perfusion imaging - laser speckle contrast analysis}

I NTRAOPERATIVE visualization and documentation of real-time vessel perfusion has become an indispensable component during neurovascular procedures. 3,4,22,27,28 While indocyanine green videoangiography or intraoperative DS angiography can be applied to evaluate vascular patency during bypass ${ }^{27,29}$ or aneurysm ${ }^{10,22}$ surgery, these procedures fail to visualize relative microcirculatory flow or tissue perfusion. Potential neurovascular indications for which a direct or indirect assessment of tissue perfusion can become critical are as follows: identification of clip-related vessel stenosis or occlusion, assessment of bypass patency, or confirmation of adequate flow replacement following revascularization for parent vessel sacrifice (hunterian ligation).

\footnotetext{
Abbreviations used in this paper: $\mathrm{CBF}=$ cerebral blood flow; $\mathrm{DS}=$ digital subtraction; EC-IC = extracranial-intracranial; ICA = internal carotid artery; LASCA = laser speckle contrast analysis; LDF = laser Doppler flowmetry; STA-MCA = superficial temporal artery-middle cerebral artery.
}

Therefore, several techniques have been described for the direct or indirect intraoperative assessment of cerebral tissue perfusion, such as thermal diffusion flowmetry, ${ }^{25}$ measurement of brain tissue $\mathrm{PO}_{2},{ }^{11,18} \mathrm{LDF},{ }^{6}$ or CT perfusion scanning. ${ }^{9}$ None of these techniques have gained widespread acceptance, however. Laser Doppler flowmetry allows one to assess changes in cortical microcirculatory flow ${ }^{19}$ with a high temporal resolution but remains spatially limited to small sampling volumes in the brain and is therefore prone to nonrepresentative measurements and sampling errors. Laser speckle contrast analysis is an advancement of the LDF concept, permitting semiquantitative imaging of tissue perfusion. It is a technique that provides a continuous map of blood flow velocities in real time over a variable scan area (full field). ${ }^{7}$ This map can be used to monitor tissue perfusion changes both noninvasively and in real time.

Here, we report on our first experience with LASCA as a tool for intraoperative noninvasive monitoring and imaging of cortical CBF. Three cases of direct surgical revascularization and intraoperative cortical blood flow mapping by LASCA are illustrated to address the feasi- 
TABLE 1: Summary of clinical and treatment-related data in 3 patients who underwent EC-IC bypass procedures*

\begin{tabular}{|c|c|c|c|c|c|}
\hline $\begin{array}{l}\text { Case } \\
\text { No. }\end{array}$ & $\begin{array}{l}\text { Age } \\
\text { (yrs), } \\
\text { Sex }\end{array}$ & Diagnosis & $\begin{array}{c}\text { Surgical } \\
\text { Bypass Procedure }\end{array}$ & Intraop LASCA Assessment & $\begin{array}{l}\text { Postop DS } \\
\text { Angiography } \\
\text { Assessment } \\
\text { (days postop) }\end{array}$ \\
\hline 1 & $57, \mathrm{M}$ & rt ICAO & STA-MCA bypass rt & $\begin{array}{l}\text { cortical CBF flux, cortical vasculature, patent bypass, baseline CBF } \\
\text { flux increase }\end{array}$ & patent bypass (10) \\
\hline 2 & $30, M$ & MMD & STA-MCA bypass It & $\begin{array}{l}\text { cortical CBF flux, cortical vasculature, patent bypass, baseline CBF } \\
\text { flux increase }\end{array}$ & patent bypass (7) \\
\hline 3 & $71, \mathrm{~F}$ & rt ICA aneurysm & RAG IF-bypass rt & $\begin{array}{l}\text { cortical CBF flux, cortical vasculature, patent bypass, adequate flow } \\
\text { replacement }\end{array}$ & patent bypass (8) \\
\hline
\end{tabular}

* ICAO = ICA occlusion; IF = intermediate flow; RAG = radial artery graft.

bility of this imaging technique as a tool for the real-time assessment of relative $\mathrm{CBF}$ in the intraoperative setting.

\section{Methods \\ Technique Used for Intraoperative LASCA}

Over the last 30 years, LDF has been established as a routine experimental and clinical tool for noninvasive monitoring of blood flow velocity, traditionally expressed through the arbitrary blood flow unit "flux." However, LDF is technically limited in that blood flow is only measured at a single point. Only if the LDF probe is not repositioned during the measurement period, blood flow changes can be measured in a comparative fashion, for example, before and after an intervention expected to cause variations in regional blood flow. The quality of these LDF flux measurements are termed "relative," since it is impossible to deduct or quantify the absolute CBF (measured in $\mathrm{ml} / 100 \mathrm{mg} / \mathrm{min}$ ) or to compare regional blood flow velocities over a large surface area. As an advancement of conventional LDF, LASCA was developed as a complementary "full-field" laser scanning technique to simultaneously obtain a map of blood flow velocity distribution over a larger surface area and provide real-time images of blood flow. Laser speckle is a random interference effect that occurs after an object is illuminated by laser light. Laser speckle contrast analysis exploits the fact that the random speckle pattern generated when tissue is illuminated by laser light changes when blood cells move within the region of interest. When there is a high level of movement (fast flow), the changing pattern becomes more blurred and the contrast in that region reduces accordingly. Therefore, low contrast is related to high flow and high contrast to low flow. ${ }^{7}$ The contrast image is processed to produce a color-coded live image (flux image: red = high flow, blue = low flow) that correlates with the blood flow velocity in the tissue.

The LASCA imager we used is a $22 \times 8 \times 23-\mathrm{cm}$ portable device (MoorFLPI, Moor Instruments) mounted on an adjustable tripod and connected to a standard laptop computer equipped with real-time data acquisition software (MoorFLPI measurement software, version 2.01, Moor Instruments). Video frame rates of flow within the microcirculation are provided at up to 25 images per second at a maximum resolution of 49,000 pixels $/ \mathrm{cm}^{2}$ (for reference, the system simultaneously records a corresponding gray scale image with an integrated chargecoupled device video camera). The manual zoom allows a variable scan area from $5 \times 7 \mathrm{~mm}$ to $8 \times 12 \mathrm{~cm}$ with a working distance of $15-45 \mathrm{~cm}$ between the scan head and the measurement site. The laser penetration depth depends on the optical properties of the laser light and the sampled tissue and is reported in the range of $500 \mu \mathrm{m}-1$ mm. ${ }^{13,17}$ Vessels can be imaged in exposed tissue such as the brain, where the vasculature courses close to the surface. To obtain a maximum spatial resolution, live images can be sampled down to $0.02 \mathrm{~Hz}$. Since this sampling rate significantly reduces temporal resolution and is not feasible for real-time evaluation of relative cortical blood flow, a sampling rate of $1 \mathrm{~Hz}$ was selected for all live imaging and single-point flux measurements. Single-point flux measurements are similar to conventional laser Doppler measurements. A region of interest is defined (flexible in size and location), and the mean flow in that region is calculated and plotted in real time. The resultant trace can be analyzed to compare flow changes before, during, and after an intervention.

\section{Patient Population}

Detailed patient characteristics are outlined in Table 1. Three EC-IC bypass procedures were performed in 2 male and 1 female patient (age range 30-71 years). Indications for EC-IC bypass surgery were flow augmentation for hemodynamic compromise due to atherosclerotic cerebrovascular disease with chronic occlusion of the ICA (Case 1) and moyamoya disease (Case 2; Fig. 1A and B upper left) as well as flow replacement for the treatment of a right-sided fusiform giant aneurysm of the ICA via parent artery sacrifice (Case 3 ). In the ischemia cases, patients underwent revascularization with a standard STA-MCA bypass. For aneurysm treatment, the patient underwent revascularization with an intermediate-flow radial artery bypass; the 71-year-old woman in this case presented with a 9-month history of progressive right-sided vision loss. Computed tomography and MR imaging demonstrated compression of the right-sided optic nerve by a large ipsilateral mass lesion, which was confirmed as a fusiform parophthalmic giant aneurysm $(>3 \mathrm{~cm})$ on DS angiography (Fig. 2D). Given the complex nature of the aneurysm, primary clipping or endovascular treatment 


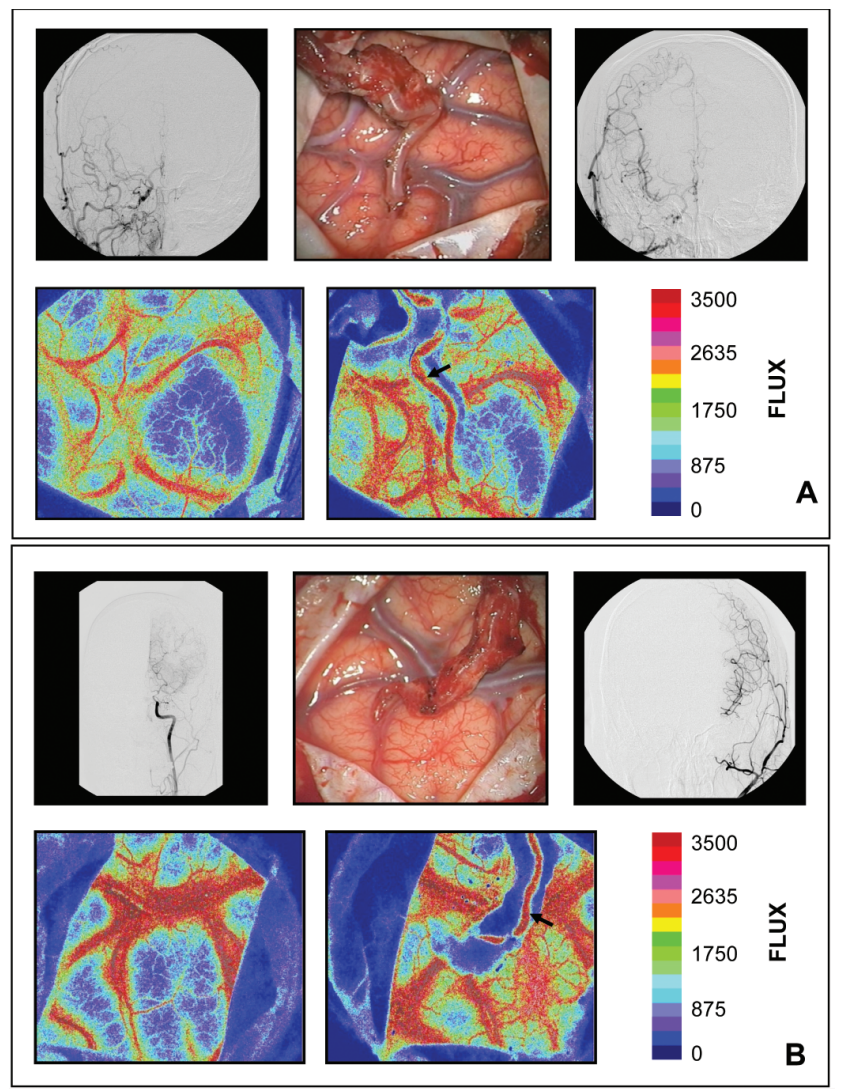

FIG. 1. Laser speckle contrast analysis of relative cortical blood flow in 2 patients with hemodynamic compromise who underwent a standard STA-MCA bypass procedure. The laser speckle pattern is visualized as a color-coded blood flow velocity and referenced in the arbitrary unit flux (range 0-3500; low flow = blue; high flow = red). Case 1. Images (A) obtained in a 57-year-old man with atherosclerotic cerebrovascular disease and right-sided ICA occlusion. Case 2. Images (B) obtained in a 30-year-old man with atypical moyamoya disease and unilateral stenosis of the left proximal ICA. Preoperative DS angiogram, anteroposterior projection (upper left), showing reduced cortical filling after hemodynamic compromise through right-sided occlusion $(A)$ and left-sided ICA stenosis (B). Intraoperative photographs (upper center) obtained after standard STA-MCA bypass procedure, demonstrating the surgical exposure site and bypass graft corresponding to the imaging field for postoperative LASCA. Postoperative DS angiograms, anteroposterior projection (upper right), of the external carotid artery demonstrating bypass graft patency. Intraoperative live laser speckle images obtained before standard STA-MCA bypass (lower left), showing color-coded LASCA visualization of cortical vasculature and CBF flux. Intraoperative live laser speckle images (lower center) obtained after completion of the anastomosis, showing a color-coded flux increase in baseline flow and visualization of the graft (arrowheads). Bar graphs (lower right) indicating color-coded arbitrary units of relative blood flow velocity in flux.

was not feasible, and trapping was determined as the therapy of choice. To next assess the risk of ischemia after ICA sacrifice, a balloon occlusion test was performed. Occlusion was associated with a moderate $\mathrm{CBF}$ decrease and insufficient collateralization of the right hemisphere via the anterior and posterior circulation. Based on this constellation, the indication for surgical revascularization before treatment of the aneurysm was given.

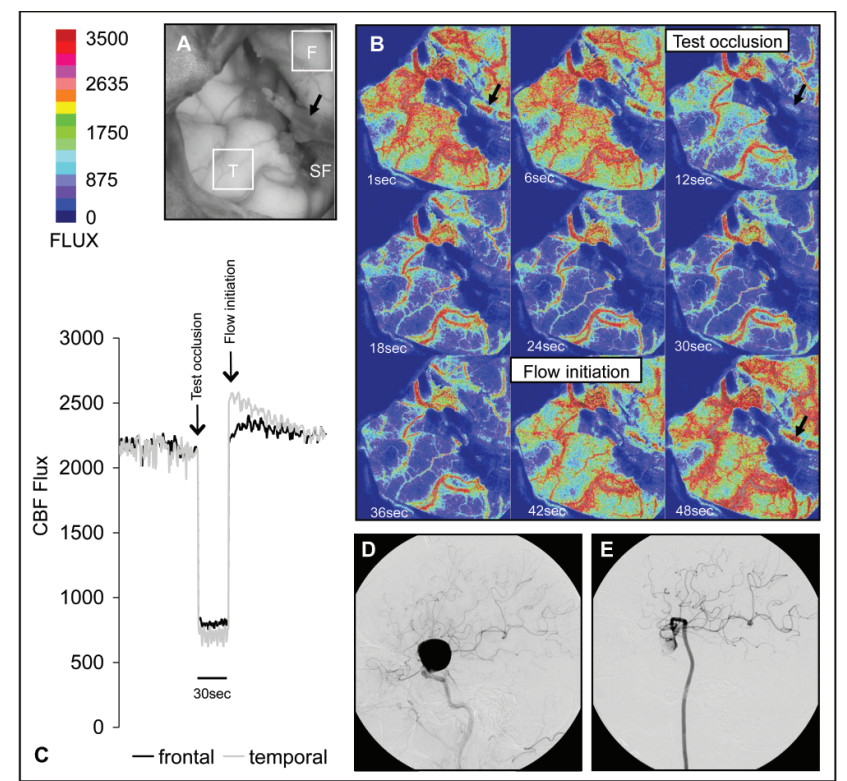

FIG. 2. Case 3. Intraoperative LASCA showing dynamic CBF changes in a 71-year-old patient with a radial artery bypass graft and proximal occlusion of the ICA for trapping of a parophthalmic giant aneurysm. A: Gray scale image of corresponding laser speckle images shown in panel $B$ with superimposed regions of interest for single-point flux measurements. Arrowhead indicates radial artery bypass graft entering the distal end of the sylvian fissure. $\mathrm{F}=$ frontal; $T=$ temporal; $\mathrm{SF}=$ sylvian fissure. $\quad$ B: Dynamic live laser speckle images depicted in 6-second intervals, illustrating the relative CBF decrease during test occlusion of the radial artery graft and proximal ICA occlusion. C: Graph showing the corresponding single-point flux measurement during temporary clipping. Arrows indicate the time point of the test occlusion and flow initiation in the graft. After clipping, there is an immediate and marked decline in $\mathrm{CBF}$ flux by $~ 65 \%$, which is maintained during the entire occlusion period of 30 seconds. Removal of the clip immediately restored perfusion and resulted in reactive hyperemia with a return to baseline perfusion within 90 seconds. D: Preoperative DS angiogram, lateral projection, of left ICA demonstrating a parophthalmic giant aneurysm. E: Postoperative DS angiogram, lateral projection, of left common carotid artery demonstrating radial artery graft patency with minimal retrograde filling of the aneurysm.

\section{Imaging Protocol}

To evaluate the general feasibility of LASCA in the intraoperative setting, live imaging was performed over the surgically exposed area for a standard STA-MCA bypass procedure ${ }^{21}$ before and after completion of the anastomosis. To document the flow contribution of the radial artery bypass after proximal occlusion of the ICA, we performed live and single-point LASCA imaging over the distal end of the sylvian fissure on completion of the anastomosis during temporary bypass occlusion and subsequent initiation of flow in the radial artery graft.

The device was centered over the surgical field $\sim 30$ $\mathrm{cm}$ above the cortical surface area. Direct illumination of the surgical field by light sources other than the laser light was avoided. The live laser speckle image with its corresponding gray scale image was visualized on the laptop screen, focused, and recorded for a period of 5 minutes. For the single-point flux measurements in Case 3, two 2 $\times 3-\mathrm{cm}$ regions of interest were positioned over cortical 
surface areas of the frontal and temporal lobes (Fig. 2A). Within these regions, the mean relative blood flow was sampled at $1 \mathrm{~Hz}$.

\section{Results}

Laser speckle live images of relative $\mathrm{CBF}$ were successfully obtained in all cases. All live image measurements were characterized by high image quality and spatial resolution. The technique permitted reliable imaging of relative blood flow in large- and small-caliber cortical vessels (Figs. 1 and 2B).

Following surgical revascularization, laser speckle imaging allowed the surgical team to judge the presence of graft flow after the completion of anastomosis (Figs. 1 and 2 arrowheads). A comparison between the intraoperative findings of LASCA and the results of early postoperative DS angiography confirmed bypass graft patency and adequate bypass function in all patients (Figs. 1 upper right panels and $2 \mathrm{E}$ ).

In cases of surgical revascularization for hemodynamic compromise, LASCA permitted visualization of an increased baseline flow after completion of the anastomosis (Fig. 1 lower panels). After radial artery bypass grafting, laser speckle and single-point imaging permitted an assessment of adequate flow contribution through the bypass graft in high spatiotemporal resolution. During test occlusion of the bypass graft after proximal vessel sacrifice, we were able to document a sudden and pronounced mean CBF flux drop from 2153.5 to 751.6 $(\sim 65 \%)$ with insufficient collateralization as previously indicated by the failed balloon occlusion test. After reinitiation of blood flow through the bypass, an immediate increase in CBF was noted with a short period of reactive hyperemia. Within 90 seconds, blood flow returned to the baseline level (Fig. 2B and C).

\section{Discussion}

We evaluated LASCA as a noninvasive technique for immediate intraoperative assessment of relative cortical blood flow. In 3 patients undergoing direct surgical revascularization, laser speckle live images of relative CBF and blood flow in large and small cortical vessels were successfully obtained. Furthermore, LASCA displayed a dynamic response capability for the sensitive detection of CBF fluctuations at a high spatial resolution.

For intraoperative monitoring of $\mathrm{CBF}$, several approaches have been pursued primarily for angiographic visualization of the cerebral vasculature or invasive assessment of CBF. Angiographic imaging techniques serve as practical tools for the evaluation of cerebral vessel patency and perfusion. While DS angiography remains the gold standard, ${ }^{4,10,23,29}$ it is invasive and associated with high financial and personal costs. Alternatively, the noninvasive visualization of brain vasculature through indocyanine green videoangiography ${ }^{22,27,28}$ permits inexpensive, high-quality imaging of the cortical vasculature. However, both techniques fail to measure relative CBF instantaneously, and the systemic administration of a contrast agent may limit technical repeatability.
On the other hand, invasive techniques allow continuous monitoring of $\mathrm{CBF}$ in localized regions. For indirect monitoring of the cerebral perfusion status, brain tissue $\mathrm{PO}_{2}$ may serve as a surrogate marker for CBF. ${ }^{26}$ Despite a low spatial and temporal resolution with no definite threshold for ischemic injury, recent studies have provided evidence for the prognostic value of brain tissue $\mathrm{PO}_{2}$ regarding outcome after severe subarachnoid hemorrhage, ${ }^{16}$ malignant ischemic stroke, ${ }^{12}$ and traumatic brain injury. ${ }^{15}$ Nevertheless, it remains a surrogate marker for the true hemodynamic situation, which is best characterized by invasive $\mathrm{CBF}$ measurements. For this purpose, invasive thermal diffusion flowmetry can be used for continuous real-time assessment of intraparenchymal blood flow, 25 comparable with measurement by xenon-enhanced CT. ${ }^{24}$

To establish noninvasive methods of intraoperative flow assessment that do not require contrast agent application, utrasonographic ${ }^{2}$ and thermal imaging techniques $^{20}$ have been investigated but remain limited by low spatial resolution and image quality. Alternatively, LDF is considered an excellent technique for instantaneous, continuous, and real-time measurements of relative $\mathrm{CBF}$ changes when determining responses to therapeutic interventions and detecting ischemic insults..$^{19}$ Despite its high temporal resolution and dynamic character, it is limited to a low spatial resolution, which hampers blood flow measurements over a wide surface area.

Laser speckle contrast analysis is a technical advancement of conventional LDF and was originally designed for the measurement of retinal blood flow. ${ }^{8}$ More recently, it has been investigated as a tool for mapping CBF responses under normal ${ }^{1,14}$ and pathophysiological ${ }^{1,5}$ experimental conditions. Monitoring the extent of spatial and temporal CBF changes by LASCA is particularly attractive for neurosurgical procedures. Access to the cortical surface is easily gained, and no further technical requirements need to be met. In our study, we experienced the handling of the LASCA device as simple, requiring minimal personnel effort. Laser speckle live imaging delivered an immediate noninvasive functional readout of relative cortical blood flow with direct assessment of tissue perfusion. Additionally, LASCA allowed visualization of blood flow in large- and small-caliber cortical vessels as well as confirmation of bypass graft patency after surgical revascularization without the application of fluorescent or radiopaque dyes. Regarding microcirculatory flow in 2 patients with hemodynamic compromise, we noted an increase in relative baseline perfusion after completion of the STA-MCA anastomosis, indicating flow augmentation provided by the bypass. In a patient who required proximal vessel sacrifice for the treatment of a complex aneurysm, we were then able to confirm an immediate and dynamic response capability of the technique for the detection of sudden perfusion changes. Laser speckle live imaging during test occlusion of the radial artery graft confirmed adequate flow replacement after subsequent flow initiation through the bypass. The high spatial and temporal resolution of LASCA for the detection of sudden changes in CBF was confirmed by additional single-point flux recordings in 2 regions of interest in the frontal and temporal lobes. 
Despite these promising preliminary results, some limitations of the technique must be addressed. First, the surgery must be interrupted for positioning of the LASCA device over the surgical field and during the measurement period. Second, LASCA has a lower tissue penetration depth compared with conventional laser Doppler imaging of $0.5-1 \mathrm{~mm}$, which may only allow measurements of directly exposed regions of the cortical surface and surrounding vasculature. Third, LASCA may indeed permit dynamic intraoperative monitoring of $\mathrm{CBF}$ variations, but at present can only be safely regarded as a qualitative measure for absolute CBF. Although CBF cannot be directly obtained in an absolute or quantitative unit $(\mathrm{ml} / 100$ $\mathrm{mg} / \mathrm{min}$ ), it was nevertheless possible to quantify regional or cortical CBF velocities in an arbitrary unit (flux) in addition to deducting relative changes in cortical blood flow as seen after test occlusion and subsequent flow initiation of an intermediate-flow bypass graft and sacrificed parent vessel. To what extent these measurements might allow a valid deduction of actual $\mathrm{CBF}$ remains under clinical and experimental investigation.

\section{Conclusions}

Laser speckle contrast analysis provided real-time and dynamic live imaging of relative cortical blood flow, flow within the cortical vasculature, and assessment of bypass graft patency with high spatial resolution and good image quality at a low cost and with minimal personnel effort. Laser speckle contrast analysis may be considered as a complementary method for intraoperative dynamic imaging of relative $\mathrm{CBF}$ and may be useful in improving the quality of neurovascular procedures.

\section{Disclosure}

This work was supported by grants from the Deutsche Forschungsgemeinschaft (DFG 323/5-1), the Bundesministerium für Bildung und Forschung (Center for Stroke Research Berlin, 01 EO 0801), and the Kompetenznetz Schlaganfall to Drs. Dreier and Vajkoczy.

\section{References}

1. Ayata C, Dunn AK, Gursoy OY, Huang Z, Boas DA, Moskowitz MA: Laser speckle flowmetry for the study of cerebrovascular physiology in normal and ischemic mouse cortex. $\mathbf{J}$ Cereb Blood Flow Metab 24:744-755, 2004

2. Badie B, Lee FT Jr, Pozniak MA, Strother CM: Intraoperative sonographic assessment of graft patency during extracranialintracranial bypass. AJNR Am J Neuroradiol 21:1457-1459, 2000

3. Bailes JE, Tantuwaya LS, Fukushima T, Schurman GW, Davis D: Intraoperative microvascular Doppler sonography in aneurysm surgery. Neurosurgery 40:965-972, 1997

4. Barrow DL, Boyer KL, Joseph GJ: Intraoperative angiography in the management of neurovascular disorders. Neurosurgery 30:153-159, 1992

5. Bolay H, Reuter U, Dunn AK, Huang Z, Boas DA, Moskowitz MA: Intrinsic brain activity triggers trigeminal meningeal afferents in a migraine model. Nat Med 8:136-142, 2002

6. Bolognese P, Miller JI, Heger IM, Milhorat TH: Laser-Doppler flowmetry in neurosurgery. J Neurosurg Anesthesiol 5:151-158, 1993
7. Briers JD: Laser Doppler, speckle and related techniques for blood perfusion mapping and imaging. Physiol Meas 22:R35-R66, 2001

8. Briers JD, Fercher AF: Retinal blood-flow visualization by means of laser speckle photography. Invest Ophthalmol Vis Sci 22:255-259, 1982

9. Chen A, Shyr MH, Chen TY, Lai HY, Lin CC, Yen PS: Dynamic CT perfusion imaging with acetazolamide challenge for evaluation of patients with unilateral cerebrovascular steno-occlusive disease. AJNR Am J Neuroradiol 27:18761881,2006

10. Chiang VL, Gailloud P, Murphy KJ, Rigamonti D, Tamargo RJ: Routine intraoperative angiography during aneurysm surgery. J Neurosurg 96:988-992, 2002

11. Dings J, Meixensberger J, Jager A, Roosen K: Clinical experience with 118 brain tissue oxygen partial pressure catheter probes. Neurosurgery 43:1082-1095, 1998

12. Dohmen C, Bosche B, Graf R, Reithmeier T, Ernestus RI, Brinker G, et al: Identification and clinical impact of impaired cerebrovascular autoregulation in patients with malignant middle cerebral artery infarction. Stroke 38:56-61, 2007

13. Dunn AK, Bolay H, Moskowitz MA, Boas DA: Dynamic imaging of cerebral blood flow using laser speckle. J Cereb Blood Flow Metab 21:195-201, 2001

14. Durduran T, Burnett MG, Yu G, Zhou C, Furuya D, Yodh $\mathrm{AG}$, et al: Spatiotemporal quantification of cerebral blood flow during functional activation in rat somatosensory cortex using laser-speckle flowmetry. J Cereb Blood Flow Metab 24:518-525, 2004

15. Jaeger M, Schuhmann MU, Soehle M, Meixensberger J: Continuous assessment of cerebrovascular autoregulation after traumatic brain injury using brain tissue oxygen pressure reactivity. Crit Care Med 34:1783-1788, 2006

16. Jaeger M, Schuhmann MU, Soehle M, Nagel C, Meixensberger J: Continuous monitoring of cerebrovascular autoregulation after subarachnoid hemorrhage by brain tissue oxygen pressure reactivity and its relation to delayed cerebral infarction. Stroke 38:981-986, 2007

17. Jakobsson A, Nilsson GE: Prediction of sampling depth and photon pathlength in laser Doppler flowmetry. Med Biol Eng Comput 31:301-307, 1993

18. Kiening KL, Unterberg AW, Bardt TF, Schneider GH, Lanksch WR: Monitoring of cerebral oxygenation in patients with severe head injuries: brain tissue PO2 versus jugular vein oxygen saturation. J Neurosurg 85:751-757, 1996

19. Kirkpatrick PJ, Smielewski P, Czosnyka M, Pickard JD: Continuous monitoring of cortical perfusion by laser Doppler flowmetry in ventilated patients with head injury. J Neurol Neurosurg Psychiatry 57:1382-1388, 1994

20. Nakagawa A, Hirano T, Uenohara H, Sato M, Kusaka Y, Shirane R, et al: Intraoperative thermal artery imaging of an EC-IC bypass in beagles with infrared camera with detectable wave-length band of 7-14 microm: possibilities as novel blood flow monitoring system. Minim Invasive Neurosurg 46:231-234, 2003

21. Pena-Tapia PG, Kemmling A, Czabanka M, Vajkoczy P, Schmiedek P: Identification of the optimal cortical target point for extracranial-intracranial bypass surgery in patients with hemodynamic cerebrovascular insufficiency. J Neurosurg 108:655-661, 2008

22. Raabe A, Beck J, Gerlach R, Zimmermann M, Seifert V: Nearinfrared indocyanine green video angiography: a new method for intraoperative assessment of vascular flow. Neurosurgery 52:132-139, 2003

23. Tang G, Cawley CM, Dion JE, Barrow DL: Intraoperative angiography during aneurysm surgery: a prospective evaluation of efficacy. J Neurosurg 96:993-999, 2002

24. Vajkoczy P, Horn P, Thome C, Munch E, Schmiedek P: Regional cerebral blood flow monitoring in the diagnosis of 


\section{N. Hecht et al.}

delayed ischemia following aneurysmal subarachnoid hemorrhage. J Neurosurg 98:1227-1234, 2003

25. Vajkoczy P, Roth H, Horn P, Lucke T, Thome C, Hubner U, et al: Continuous monitoring of regional cerebral blood flow: experimental and clinical validation of a novel thermal diffusion microprobe. J Neurosurg 93:265-274, 2000

26. Valadka AB, Hlatky R, Furuya Y, Robertson CS: Brain tissue PO2: correlation with cerebral blood flow. Acta Neurochir Suppl (Wien) 81:299-301, 2002

27. Woitzik J, Horn P, Vajkoczy P, Schmiedek P: Intraoperative control of extracranial-intracranial bypass patency by nearinfrared indocyanine green videoangiography. J Neurosurg 102:692-698, 2005

28. Woitzik J, Pena-Tapia PG, Schneider UC, Vajkoczy P, Thome
C: Cortical perfusion measurement by indocyanine-green videoangiography in patients undergoing hemicraniectomy for malignant stroke. Stroke 37:1549-1551, 2006

29. Yanaka K, Fujita K, Noguchi S, Matsumaru Y, Asakawa H, Anno I, et al: Intraoperative angiographic assessment of graft patency during extracranial-intracranial bypass procedures. Neurol Med Chir (Tokyo) 43:509-513, 2003

Manuscript submitted June 15, 2009.

Accepted August 19, 2009.

Address correspondence to: Peter Vajkoczy, M.D., CharitéUniversitätsmedizin Berlin, Department of Neurosurgery, Augustenburgerplatz 1, 13353 Berlin, Germany. email: peter.vajkoczy@ charite.de. 